\title{
Review Article \\ Recent Advances in Treatments of Primary Focal Segmental Glomerulosclerosis in Children
}

\author{
Kyoung Hee Han ${ }^{1}$ and Seong Heon Kim ${ }^{2,3}$ \\ ${ }^{1}$ Department of Pediatrics, Jeju National University School of Medicine, Aran 13gil 15, Jeju-si, \\ Jeju Special Self-Governing Province 63241, Republic of Korea \\ ${ }^{2}$ Department of Pediatrics, Pusan National University Children's Hospital, 20 Geumo-ro, Mulgeum-eup, Yangsan-si, \\ Gyeongsangnam-do 50612, Republic of Korea \\ ${ }^{3}$ Research Institute for Convergence of Biomedical Science and Technology, Pusan National University Yangsan Hospital, \\ 20 Geumo-ro, Mulgeum-eup, Yangsan-si, Gyeongsangnam-do 50612, Republic of Korea
}

Correspondence should be addressed to Seong Heon Kim; pedksh@gmail.com

Received 22 December 2015; Revised 21 March 2016; Accepted 30 March 2016

Academic Editor: Jun Oh

Copyright (C) 2016 K. H. Han and S. H. Kim. This is an open access article distributed under the Creative Commons Attribution License, which permits unrestricted use, distribution, and reproduction in any medium, provided the original work is properly cited.

Focal segmental glomerulosclerosis (FSGS) is a nephrotic syndrome. Up to around $80 \%$ of cases of primary FSGS are resistant to steroid treatment. A large proportion of patients with steroid-resistant FSGS progress to end-stage renal disease. The purpose of treatment is to obtain a complete remission of proteinuria, a necessary step that precedes improved renal survival and reduces the risk of progression to chronic kidney disease. When this is not possible, the secondary goal is a partial remission of proteinuria. Reduction or remission of proteinuria is the most important factor predictive of renal survival. We will review the current updated strategies for treatment of primary FSGS in children, including traditional therapies consisting of corticosteroids and calcineurin inhibitors and novel therapies such as rituximab, abatacept, adalimumab, and fresolimumab.

\section{Introduction}

Idiopathic nephrotic syndrome (INS) is a primary renal disorder defined by the three signs of proteinuria, hypoalbuminemia, and edema. Histopathological categories of INS include minimal change disease, focal segmental glomerulosclerosis (FSGS), and diffuse mesangial proliferation [1]. Based on the response to steroid treatment, two types of INS can be characterized: steroid-sensitive nephrotic syndrome (SSNS), in which the proteinuria resolves, and steroidresistant nephrotic syndrome (SRNS), in which the proteinuria does not resolve [1]. Most patients with minimal change disease respond well to steroid treatment and, currently, renal biopsy is not routinely performed in patients with SSNS. Therefore, the terminology of minimal change disease has become synonymous with SSNS [1]. However, genetic forms of nephrotic syndrome have been emphasized since the discovery of causative mutations in cases with infantile and juvenile SRNS and familial nephrotic syndrome [1]. Histologic findings in the kidney reveal FSGS in most patients with both syndromic and nonsyndromic forms of SRNS [1]. Therefore, it is important to rule out genetic forms of FSGS beforehand to avoid trying ineffective therapies.

A report of the International Study of Kidney Disease in Children (ISKDC) demonstrated that, among patients who were initial steroid responders and those who were not, $3 \%$ and $47.5 \%$ had FSGS, respectively [2]. Up to around $80 \%$ of cases of primary FSGS are resistant to steroids [3]. A large proportion of patients with steroid-resistant FSGS progress to end-stage renal disease [4]. Therefore, nephrologists are challenged and are struggling to treat patients with FSGS. We will review current strategies for medical treatment of primary FSGS in children, omitting any discussion of genetic forms or relapses of FSGS after kidney transplantation. 


\section{Corticosteroids}

Steroid therapy is the most basic and standard treatment [1]. Initial steroid therapy is with oral prednisone at a dose of $60 \mathrm{mg} / \mathrm{m}^{2}$ or $2 \mathrm{mg} / \mathrm{kg}$ per day with a maximum dose of $60 \mathrm{mg}$ per day for 4 to 6 weeks followed by $40 \mathrm{mg} / \mathrm{m}^{2}$ or $1.5 \mathrm{mg} / \mathrm{kg}$ every other day for 4 to 6 weeks $[2,5,6]$. Ehrich and Brodehl reported the advantages of a 12-week compared to an 8-week steroid treatment protocol for an initial attack of INS [7]. The cumulative rate of patients with sustained remissions after 2 years was significantly higher after the 12-week course than after the 8-week treatment [7]. Several comparative studies have reported that a longer duration of steroid treatment (3-7 months) after an initial 4-8-week daily steroid treatment followed by an alternative protocol significantly reduced the relapse rate compared with the 8-week protocol [7-10]. An alternative treatment for patients who do not achieve remission of nephrotic syndrome after the initial 4 weeks is to administer an intravenous pulse of methylprednisolone, $30 \mathrm{mg} / \mathrm{kg}$ per dose, with a maximum of $1 \mathrm{~g}$ every other day for 2 weeks $[1,11]$. Twothirds of initial steroid nonresponders eventually became responders with a 2-week course of methylprednisolone [12].

The "Mendoza" protocol is an aggressive treatment strategy based on high-dose methylprednisolone pulse therapy and oral prednisolone, with or without an alkylating agent, administered for a total of 82 weeks (Table 1) [4, 12]. When this protocol was used to treat cases of SRNS, approximately $65 \%$ went into complete remission and only $25 \%$ progressed to chronic kidney disease [4]. However, prolonged steroid treatment for nephrotic syndrome causes significant side effects, including growth impairment, obesity, hypertension, cataracts, osteoporosis, immune suppression, diabetes mellitus, psychosis, hirsutism, and striae [11].

\section{Cyclophosphamide}

Treatment with a 12-week course of cyclophosphamide at a single dose of $2 \mathrm{mg} / \mathrm{kg}$ per day with a maximum cumulative dose of $168 \mathrm{mg} / \mathrm{kg}$ reduced the rate of relapse in steroid dependent nephrotic syndrome (SDNS) $[6,13]$. A longer remission was observed when cyclophosphamide treatment was used in combination with steroids compared to cyclophosphamide given alone for SDNS [14]. There is little data indicating that cyclophosphamide is effective in SRNS with FSGS and updated version of the Kidney Disease: Improving Global Outcomes (KDIGO) guidelines (http://kdigo.org/home/glomerulonephritis-gn/) suggests that cyclophosphamide should not be given to children with SRNS [1517]. However, renal failure progresses less frequently in SRNS patients who are partial responders to cyclophosphamide $[18,19]$.

Cyclophosphamide can be used as monthly intravenous boluses at a dose of $500 \mathrm{mg} / \mathrm{m}^{2}$ for 6 months for infrequently relapsing nephrotic syndrome [20]. There are divergent opinions about intravenous pulse cyclophosphamide for steroid-resistant FSGS [21]. However, intravenous pulse
TABLE 1: The "Mendoza" protocol for steroid-resistant nephrotic syndrome in children.

\begin{tabular}{lcc}
\hline Week & Methylprednisolone, $30 \mathrm{mg} / \mathrm{kg}$ & Oral prednisone \\
\hline $1-2$ & Every other day, 6 doses & None \\
$3-10$ & Every week, 8 doses & 2 mg/kg every other day \\
$11-18$ & Every 2 weeks, 4 doses & With/without taper \\
$19-50$ & Every 4 weeks, 8 doses & Slow taper \\
$51-82$ & Every 8 weeks, 4 doses & Slow taper \\
\hline
\end{tabular}

cyclophosphamide has been considered as an adjunctive therapy for steroid-resistant FSGS [22].

\section{Calcineurin Inhibitors}

4.1. Cyclosporine. Cyclosporine reduces the relapse rate by $80 \%$ in patients with SDNS [23]. However, the patients tend to become cyclosporine dependent just as they did with steroids [1]. The remission rate is significantly higher when the therapeutic dosage of cyclosporine is $4-6 \mathrm{mg} / \mathrm{kg} / \mathrm{day}$ in two divided doses to maintain a trough level between 60 and $80 \mathrm{ng} / \mathrm{mL}$ in patients with SDNS [1, 6, 24]. Some research has demonstrated that cyclosporine in combination with steroids is significantly effective in reducing the relapse rate in patients with SRNS $[25,26]$. Another study showed that prolonged combination therapy with cyclosporine and steroids, including intravenous pulses of methylprednisolone, maintained the remission rate at $84 \%$ for patients with SRNS [27]. Although relapses were frequent, long-term cyclosporine treatment in children with SRNS reduced the progression of chronic kidney disease by reducing proteinuria $[28,29]$. Therefore, the Canadian Society of Nephrology (CSN) recently recommended using a calcineurin inhibitor, either cyclosporine or tacrolimus, following standard therapy with steroids for children with SRNS [6]. In recent years, however, cyclosporine has been found not to work in all FSGS patients because patients with a hereditary background of the disease rarely benefit from cyclosporine therapy [30, 31]. Side effects of cyclosporine include hypertrichosis, gum hypertrophy, hypertension, and nephrotoxicity [11].

4.2. Tacrolimus. Tacrolimus has recently been highlighted as an alternative to cyclosporine for SRNS [11]. Tacrolimus at a dose of $0.1-0.2 \mathrm{mg} / \mathrm{kg}$ per day divided into two doses was effective and well tolerated in children with SRNS, with a complete remission rate of $81 \%[32,33]$. A target trough level of $5-7 \mathrm{ng} / \mathrm{mL}$ should be recommended for SRNS [6, 34]. Cosmetic side effects such as hypertrichosis and gum hypertrophy are not seen with tacrolimus, but any difference between cyclosporine and tacrolimus in the incidence of nephrotoxicity has not been proven [11]. Other side effects, including tremor, arterial hypertension, and diabetes mellitus, can occur. Recently, the efficacy of multidrug therapy consisting of tacrolimus, mycophenolate mofetil (MMF), mizoribine, or leflunomide in combination with steroids for children with SRNS has been reported [35, 36]. While the relapse rate was significantly decreased after multidrug 
therapy in children with SRNS, long-term safety has not yet been proven.

\section{Mycophenolate Mofetil}

The CSN does not recommend the widespread use of MMF due to limited cost-effectiveness [6]. However, a retrospective study in children with nephrotic syndrome reported that the combined rate of complete and partial remission for MMF was $67 \%$ in SRNS, and for refractory cases, combination therapy with MMF, tacrolimus, and steroids yielded a $75 \%$ response rate [37]. Compared to tacrolimus, the response rate for MMF was not superior, but MMF has milder side effects than calcineurin inhibitors [11]. MMF was not inferior to calcineurin inhibitors in preventing relapses but only if administered in high doses [38]. MMF can be an alternative agent in patients with adverse effects due to calcineurin inhibitors $[1,6]$. MMF can be given at a dose of $1200 \mathrm{mg} / \mathrm{m}^{2}$ per day divided into two doses. Area under the concentration-time curve (AUC) measurements are essential in the use of this drug. Commonly reported adverse effects of MMF include metabolic acidosis, infection, diarrhea, abdominal pain, and hyperlipidemia.

\section{Mizoribine}

Mizoribine inhibits inosine monophosphate synthetase and guanosine monophosphate synthetase, resulting in the inhibition of DNA synthesis and cell division. Mizoribine has fewer side effects than azathioprine, which also inhibits an enzyme required for DNA synthesis $[39,40]$. Although the mechanism of action for mizoribine is similar to the more widely used azathioprine and MMF, there is little data as to whether it is effective in maintaining remission in nephrotic syndrome [41]. However, there are some case series reporting successful treatment with combination therapy using mizoribine, tacrolimus, or plasmapheresis for children with refractory nephrotic syndrome in Japan [35, 42]. Mizoribine can be administered at a dose of $3 \mathrm{mg} / \mathrm{kg}$ once daily before breakfast [35].

\section{Renin-Angiotensin System Blockade}

The KDIGO Work Group recommended renin-angiotensin system (RAS) inhibition with angiotensin converting enzyme inhibitor (ACE-I) or angiotensin II receptor blockers (ARBs) for children with SRNS [6]. RAS blockade combined with MMF was markedly effective in children and young adults with steroid-resistant FSGS [43]. A comparative study of ACE-I and calcium channel blockers in SRNS demonstrated that both ramipril and verapamil reduced proteinuria in patients with SRNS [44]. Another randomized trial of 45 children (10 with FSGS) with normotensive SRNS suggested that fosinopril significantly reduced proteinuria [45]. RAS blockade is especially effective for those patients with chronic renal insufficiency. Monitoring adverse effects of RAS inhibition, notably hyperkalemia and reduction of glomerular filtration rate, is necessary during treatment [11].

\section{Galactose}

Oral galactose, a monosaccharide sugar, inhibits the circulating permeability activity that is the cause of FSGS [46]. However, currently, there is little evidence that it improves proteinuria in children with FSGS [47]. There are only a few case reports about partial remission after oral galactose therapy at a dose of $0.2 \mathrm{~g} / \mathrm{kg}$ twice a day as a nontoxic and adjunctive agent for SRNS [48, 49].

\section{Rituximab}

Rituximab is a chimeric monoclonal antibody that inhibits CD20-mediated B lymphocytes [11]. It has been used in hematologic malignancies such as B cell non-Hodgkin lymphoma [50]. Rituximab is administered by two to four intravenous infusions at a dose of $375 \mathrm{mg} / \mathrm{m}^{2}$ weekly or biweekly [41]. There are several reports of rituximab therapy being an effective steroid-sparing agent for children with SDNS [51, 52] or SRNS [50, 53, 54]. Rituximab and other immunosuppressants such as cyclosporine or MMF combination therapy for SRNS have been reported to be successful in decreasing the relapse rate $[55,56]$. However, some patients with SDNS become rituximab dependent as they do with steroids or calcineurin inhibitors [51]. Anti-rituximab autoantibodies may be a concern after repetitive infusion of rituximab [57]. Therefore, the presence of anti-rituximab autoantibodies should be monitored if a severe infusion reaction occurs and $B$ cell depletion is not observed despite rituximab therapy [57, 58].

\section{Synthetic Adrenocorticotropin Analog}

Injection with adrenocorticotropin (ACTH) was used many decades ago as a therapeutic agent for children with nephrotic syndrome, but it has been replaced by cheaper oral steroids [59]. Response to ACTH among patients with FSGS is about $30 \%$, but ACTH gel may be an alternative treatment option for some patients with SRNS [60, 61].

\section{Abatacept}

Abatacept (cytotoxic T-lymphocyte-associated antigen 4immunoglobulin fusion protein [CTLA-4-Ig]) is an inhibitor of the T-cell costimulatory molecule B7-1 (CD80) [62]. It is currently approved for the treatment of rheumatoid arthritis. Podocyte B7-1 expression was found in patients with proteinuric kidney [62]. Yu and colleagues reported five patients with FSGS, four patients with rituximab-resistant recurrent FSGS, and one patient with steroid-resistant FSGS, whose high proteinuria resolved after abatacept treatment [62]. The authors explained that abatacept may attenuate $\beta 1$-integrin activation in podocytes and decrease proteinuria in patients with B7-1-positive glomerular disease [62]. 


\section{Adalimumab}

Adalimumab is a human monoclonal antibody directed against tumor necrosis factor- $\alpha$ (TNF- $\alpha$ ), which triggers an autoimmune response [61]. The Novel Therapies for Resistant FSGS (FONT) Study Group conducted a phase I trial of adalimumab in FSGS [63]. Adalimumab was injected subcutaneously every 2 weeks at a dose of $24 \mathrm{mg} / \mathrm{m}^{2}$ with a maximum of $40 \mathrm{mg}$ for 16 weeks (total, nine doses) in 10 patients with resistant FSGS [63]. Adalimumab was well tolerated with no serious side effects and 4 out of 10 patients with proteinuria had it decreased by more than 50\% [63]. The FONT Study Group formed the basis for a phase 2 study of adalimumab in resistant FSGS [63].

\section{Fresolimumab}

Fibrosis represents the final common pathway of glomerular damage in FSGS, leading to chronic kidney disease. The experience with a wide range of antifibrotic agents was summarized in a recent review article [61]. Fresolimumab is a recombinant, fully human monoclonal antibody and inhibits the activity of all isoforms of transforming growth factor (TGF- $\beta$ ) [64]. Trachtman and colleagues conducted a phase 1, single-dose study of fresolimumab in adults with treatment-resistant FSGS [64]. They reported one case of complete remission and two cases of partial remission of proteinuria among a total of 16 patients with resistant FSGS. Further studies are needed to confirm the efficacy of fresolimumab for the treatment of resistant FSGS.

\section{Conclusions}

FSGS is a heterogeneous disorder and response to treatment is individually diverse. SRNS is an indication for a renal biopsy and genetic study. If the renal biopsy shows FSGS and if no pathogenic mutations in podocyte genes can be identified, efforts to minimize drug toxicity are important in pediatric FSGS. However, nephrologists still depend on traditional treatment approaches based on glucocorticoids plus calcineurin inhibitors. A steroid-based combination therapy with one or two drugs such as calcineurin inhibitors, MMF, or RAS blockade for refractory cases can induce partial remission of proteinuria. Severe FSGS cases experiencing treatment toxicities may try novel therapies such as rituximab, abatacept, adalimumab, or fresolimumab. In the future, further investigations of the disease mechanism will assist in the introduction of novel targeted individual approaches for treating FSGS.

\section{Disclosure}

The authors have no relevant affiliations or financial involvement with any organization or entity with a financial interest in or financial conflict with the subject matter or materials discussed in the paper.

\section{Competing Interests}

The authors have no potential competing interests to disclose.

\section{References}

[1] E. Avner, W. Harmon, P. Niaudet, and N. Yoshikawa, Pediatric Nephrology, vol. 1, Springer, 6th edition, 2009.

[2] A Report of the International Study of Kidney Disease in Children, "The primary nephrotic syndrome in children. Identification of patients with minimal change nephrotic syndrome from initial response to prednisone," The Journal of Pediatrics, vol. 98, no. 4, pp. 561-564, 1981.

[3] S. M. Korbet, M. M. Schwartz, and E. J. Lewis, "Primary focal segmental glomerulosclerosis: clinical course and response to therapy," American Journal of Kidney Diseases, vol. 23, no. 6, pp. 773-783, 1994.

[4] B. M. Tune, R. Kirpekar, R. K. Sibley, V. M. Reznik, W. R. Griswold, and S. A. Mendoza, "Intravenous methylprednisolone and oral alkylating agent therapy of prednisone-resistant pediatric focal segmental glomerulosclerosis: a long-term follow-up," Clinical Nephrology, vol. 43, no. 2, pp. 84-88, 1995.

[5] "Alternate-day versus intermittent prednisone in frequently relapsing nephrotic syndrome. A report of "Arbetsgemeinschaft für Pädiatrische Nephrologie"," The Lancet, vol. 1, no. 8113, pp. 401-403, 1979.

[6] S. Samuel, M. Bitzan, M. Zappitelli et al., "Canadian society of nephrology commentary on the 2012 KDIGO clinical practice guideline for glomerulonephritis: management of nephrotic syndrome in children," American Journal of Kidney Diseases, vol. 63, no. 3, pp. 354-362, 2014.

[7] J. H. H. Ehrich and J. Brodehl, "Long versus standard prednisone therapy for initial treatment of idiopathic nephrotic syndrome in children. Arbeitsgemeinschaft fur Padiatrische Nephrologie," European Journal of Pediatrics, vol. 152, no. 4, pp. 357-361, 1993.

[8] A. Bagga, P. Hari, and R. N. Srivastava, "Prolonged versus standard prednisolone therapy for initial episode of nephrotic syndrome," Pediatric Nephrology, vol. 13, no. 9, pp. 824-827, 1999.

[9] J. Ksiazek and T. Wyszynska, "Short versus long initial prednisone treatment in steroid-sensitive nephrotic syndrome in children," Acta Paediatrica, vol. 84, no. 8, pp. 889-893, 1995.

[10] N. Ueda, M. Chihara, S. Kawaguchi et al., "Intermittent versus long-term tapering prednisolone for initial therapy in children with idiopathic nephrotic syndrome," The Journal of Pediatrics, vol. 112, no. 1, pp. 122-126, 1988.

[11] C. B. Sethna and D. S. Gipson, "Treatment of FSGS in children," Advances in Chronic Kidney Disease, vol. 21, no. 2, pp. 194-199, 2014.

[12] A. Meyrier, "Treatment of primary focal segmental glomerulosclerosis," Nephrology Dialysis Transplantation, vol. 14, supplement 3, pp. 74-78, 1999.

[13] B. S. Oemar and J. Brodehl, "Cyclophosphamide treatment of steroid dependent nephrotic syndrome: comparison of eight week with 12 week course. Report of Arbeitsgemeinschaft fur Padiatrische Nephrologie," Archives of Disease in Childhood, vol. 62, no. 11, pp. 1102-1106, 1987.

[14] "Prospective, controlled trial of cyclophosphamide therapy in children with nephrotic syndrome. Report of the International study of Kidney Disease in Children," The Lancet, vol. 2, no. 7878, pp. 423-427, 1974. 
[15] P. Tarshish, J. N. Tobin, J. Bernstein, and C. M. Edelmann Jr., "Cyclophosphamide does not benefit patients with focal segmental glomerulosclerosis. A report of the International Study of Kidney Disease in Children," Pediatric Nephrology, vol. 10, no. 5, pp. 590-593, 1996.

[16] R. H. White and E. F. Glasgow, "Focal glomerulosclerosis-a progressive lesion associated with steroid-resistant nephrotic syndrome," Archives of Disease in Childhood, vol. 46, no. 250, pp. 877-878, 1971.

[17] "Chapter 4: steroid-resistant nephrotic syndrome in children," Kidney International Supplements, vol. 2, no. 2, pp. 172-176, 2012.

[18] D. F. Geary, M. Farine, P. Thorner, and R. Baumal, "Response to cyclophosphamide in steroid-resistant focal segmental glomerulosclerosis: a reappraisal," Clinical Nephrology, vol. 22, no. 3, pp. 109-113, 1984.

[19] A. Bergstrand, I. Bollgren, A. Samuelsson, T. Törnroth, J. Wasserman, and J. Winberg, "Idiopathic nephrotic syndrome of childhood: cyclophosphamide induced conversion from steroid refractory to highly steroid sensitive disease," Clinical Nephrology, vol. 1, no. 5, pp. 302-306, 1973.

[20] S. Gulati, S. Pokhariyal, R. K. Sharma et al., "Pulse cyclophosphamide therapy in frequently relapsing nephrotic syndrome," Nephrology Dialysis Transplantation, vol. 16, no. 10, pp. 20132017, 2001.

[21] H. O. Alshaya, J. A. Al-Maghrabi, and J. A. Kari, "Intravenous pulse cyclophosphamide-is it effective in children with steroid-resistant nephrotic syndrome?" Pediatric Nephrology, vol. 18, no. 11, pp. 1143-1146, 2003.

[22] W. P. Rennert, U. K. Kala, D. Jacobs, S. Goetsch, and S. Verhaart, "Pulse cyclophosphamide for steroid-resistant focal segmental glomeruloscleuosis," Pediatric Nephrology, vol. 13, no. 2, pp. 113116, 1999.

[23] P. Niaudet and R. Habib, "Cyclosporine in the treatment of idiopathic nephrosis," Journal of the American Society of Nephrology, vol. 5, no. 4, pp. 1049-1056, 1994.

[24] K. Ishikura, M. Ikeda, S. Hattori et al., "Effective and safe treatment with cyclosporine in nephrotic children: a prospective, randomized multicenter trial," Kidney International, vol. 73, no. 10, pp. 1167-1173, 2008.

[25] "Safety and tolerability of cyclosporin A (Sandimmun) in idiopathic nephrotic syndrome. Collaborative Study Group of Sandimmun in Nephrotic Syndrome," Clinical Nephrology, vol. 35, supplement 1, pp. S48-S60, 1991.

[26] P. Niaudet, "Treatment of childhood steroid-resistant idiopathic nephrosis with a combination of cyclosporine and prednisone," The Journal of Pediatrics, vol. 125, no. 6, part 1, pp. 981-986, 1994.

[27] J. H. H. Ehrich, C. Geerlings, M. Zivicnjak, D. Franke, H. Geerlings, and J. Gellermann, "Steroid-resistant idiopathic childhood nephrosis: overdiagnosed and undertreated," Nephrology Dialysis Transplantation, vol. 22, no. 8, pp. 2183-2193, 2007.

[28] E. Ingulli, A. Singh, N. Baqi, H. Ahmad, S. Moazami, and A. Tejani, "Aggressive, long-term cyclosporine therapy for steroidresistant focal segmental glomerulosclerosis," Journal of the American Society of Nephrology, vol. 5, no. 10, pp. 1820-1825, 1995.

[29] D. C. Cattran, G. B. Appel, L. A. Hebert et al., "A randomized trial of cyclosporine in patients with steroid-resistant focal segmental glomerulosclerosis," Kidney International, vol. 56, no. 6, pp. 2220-2226, 1999.

[30] A. K. Buscher, B. B. Beck, A. Melk et al., "Rapid response to cyclosporin A and favorable renal outcome in nongenetic versus genetic steroid-resistant nephrotic syndrome," Clinical Journal of the American Society of Nephrology, vol. 11, no. 2, pp. 245-253, 2016.

[31] A. K. Büscher, B. Kranz, R. Büscher et al., "Immunosuppression and renal outcome in congenital and pediatric steroid-resistant nephrotic syndrome," Clinical Journal of the American Society of Nephrology, vol. 5, no. 11, pp. 2075-2084, 2010.

[32] K. Loeffler, M. Gowrishankar, and V. Yiu, “Tacrolimus therapy in pediatric patients with treatment-resistant nephrotic syndrome," Pediatric Nephrology, vol. 19, no. 3, pp. 281-287, 2004.

[33] A. Jahan, R. Prabha, S. Chaturvedi, B. Mathew, D. Fleming, and I. Agarwal, "Clinical efficacy and pharmacokinetics of tacrolimus in children with steroid-resistant nephrotic syndrome," Pediatric Nephrology, vol. 30, no. 11, pp. 1961-1967, 2015.

[34] A. Gulati, A. Sinha, A. Gupta et al., "Treatment with tacrolimus and prednisolone is preferable to intravenous cyclophosphamide as the initial therapy for children with steroid-resistant nephrotic syndrome," Kidney International, vol. 82, no. 10, pp. 1130-1135, 2012.

[35] T. Aizawa-Yashiro, K. Tsuruga, S. Watanabe, E. Oki, E. Ito, and H. Tanaka, "Novel multidrug therapy for children with cyclosporine-resistant or -intolerant nephrotic syndrome," Pediatric Nephrology, vol. 26, no. 8, pp. 1255-1261, 2011.

[36] B. Wu, J. Mao, H. Shen et al., "Triple immunosuppressive therapy in steroid-resistant nephrotic syndrome children with tacrolimus resistance or tacrolimus sensitivity but frequently relapsing," Nephrology, vol. 20, no. 1, pp. 18-24, 2015.

[37] J. Kim, N. Patnaik, N. Chorny, R. Frank, L. Infante, and C. Sethna, "Second-line immunosuppressive treatment of childhood nephrotic syndrome: a single-center experience," Nephron Extra, vol. 4, no. 1, pp. 8-17, 2014.

[38] D. S. Gipson, H. Trachtman, F. J. Kaskel et al., "Clinical trial of focal segmental glomerulosclerosis in children and young adults," Kidney International, vol. 80, no. 8, pp. 868-878, 2011.

[39] J. S. Maltzman and G. A. Koretzky, "Azathioprine: old drug, new actions," The Journal of Clinical Investigation, vol. 111, no. 8, pp. 1122-1124, 2003.

[40] R. Kusaba, O. Otubo, H. Sugimoto et al., "Immunosuppressive effect of bredinin in the management of patients with renal transplantation," Proceedings of the European Dialysis and Transplant Association, vol. 18, pp. 420-425, 1981.

[41] M. van Husen and M. J. Kemper, "New therapies in steroidsensitive and steroid-resistant idiopathic nephrotic syndrome," Pediatric Nephrology, vol. 26, no. 6, pp. 881-892, 2011.

[42] T. Imaizumi, Y. Kawasaki, H. Matsuura et al., "Efficacy of steroid pulse, plasmapheresis, and mizoribine in a patient with focal segmental glomerulosclerosis," Pediatric Nephrology, vol. 22, no. 8, pp. 1215-1218, 2007.

[43] B. Montané, C. Abitbol, J. Chandar, J. Strauss, and G. Zilleruelo, "Novel therapy of focal glomerulosclerosis with mycophenolate and angiotensin blockade," Pediatric Nephrology, vol. 18, no. 8, pp. 772-777, 2003.

[44] N. S. Kumar, A. K. Singh, R. N. Mishra, and J. Prakash, "Comparative study of angiotensin converting enzyme inhibitor and calcium channel blocker in the treatment of steroidresistant idiopathic nephrotic syndrome," Journal of Association of Physicians of India, vol. 52, pp. 454-458, 2004.

[45] Z. Yi, Z. Li, X.-C. Wu, Q.-N. He, X.-Q. Dang, and X.-J. He, "Effect of fosinopril in children with steroid-resistant idiopathic nephrotic syndrome," Pediatric Nephrology, vol. 21, no. 7, pp. 967-972, 2006. 
[46] V. J. Savin, E. T. McCarthy, R. Sharma, D. Charba, and M. Sharma, "Galactose binds to focal segmental glomerulosclerosis permeability factor and inhibits its activity," Translational Research, vol. 151, no. 6, pp. 288-292, 2008.

[47] K. Sgambat, M. Banks, and A. Moudgil, "Effect of galactose on glomerular permeability and proteinuria in steroid-resistant nephrotic syndrome," Pediatric Nephrology, vol. 28, no. 11, pp. 2131-2135, 2013.

[48] M. Kopač, A. Meglič, and R. R. Rus, "Partial remission of resistant nephrotic syndrome after oral galactose therapy," Therapeutic Apheresis and Dialysis, vol. 15, no. 3, pp. 269-272, 2011.

[49] E. De Smet, J.-P. Rioux, H. Ammann, C. Déziel, and S. Quérin, "FSGS permeability factor-associated nephrotic syndrome: remission after oral galactose therapy," Nephrology Dialysis Transplantation, vol. 24, no. 9, pp. 2938-2940, 2009.

[50] J. A. Kari, S. M. El-Morshedy, S. El-Desoky, H. O. Alshaya, K. A. Rahim, and B. M. Edrees, "Rituximab for refractory cases of childhood nephrotic syndrome," Pediatric Nephrology, vol. 26, no. 5, pp. 733-737, 2011.

[51] P. Ravani, A. Bonanni, R. Rossi, G. Caridi, and G. M. Ghiggeri, "Anti-CD20 antibodies for idiopathic nephrotic syndrome in children," Clinical Journal of the American Society of Nephrology, vol. 11, no. 4, pp. 710-720, 2016.

[52] M. J. Kemper, A. Lehnhardt, A. Zawischa, and J. Oh, "Is rituximab effective in childhood nephrotic syndrome? Yes and no," Pediatric Nephrology, vol. 29, no. 8, pp. 1305-1311, 2014.

[53] L. Sun, H. Xu, Q. Shen et al., "Efficacy of rituximab therapy in children with refractory nephrotic syndrome: a prospective observational study in Shanghai," World Journal of Pediatrics, vol. 10, no. 1, pp. 59-63, 2014.

[54] M. D. Pescovitz, B. K. Book, and R. A. Sidner, "Resolution of recurrent focal segmental glomerulosclerosis proteinuria after rituximab treatment," The New England Journal of Medicine, vol. 354, no. 18, pp. 1961-1963, 2006.

[55] K. Suyama, Y. Kawasaki, K. Miyazaki et al., "Rituximab and lowdose cyclosporine combination therapy for steroid-resistant FSGS," Pediatrics International, vol. 58, no. 3, pp. 219-223, 2016.

[56] B. Basu, T. K. S. Mahapatra, and N. Mondal, "Mycophenolate mofetil following rituximab in children with steroid-resistant nephrotic syndrome," Pediatrics, vol. 136, no. 1, pp. e132-e139, 2015.

[57] H. G. Kang and H. I. Cheong, "Nephrotic syndrome: what's new, what's hot?" Korean Journal of Pediatrics, vol. 58, no. 8, pp. 275282, 2015.

[58] Y. H. Ahn, H. G. Kang, J. M. Lee, H. J. Choi, I.-S. Ha, and H. I. Cheong, "Development of antirituximab antibodies in children with nephrotic syndrome," Pediatric Nephrology, vol. 29, no. 8, pp. 1461-1464, 2014.

[59] T. Mittal, P. Dedhia, P. Roy-Chaudhury et al., "Complete remission of post-transplantation recurrence of focal segmental glomerulosclerosis with the use of adrenocorticotrophic hormone gel: case report," Transplantation Proceedings, vol. 47, no. 7, pp. 2219-2222, 2015.

[60] J. Hogan, A. S. Bomback, K. Mehta et al., "Treatment of idiopathic FSGS with adrenocorticotropic hormone gel," Clinical Journal of the American Society of Nephrology, vol. 8, no. 12, pp. 2072-2081, 2013.

[61] L. Malaga-Dieguez, D. Bouhassira, D. Gipson, and H. Trachtman, "Novel therapies for FSGS: preclinical and clinical studies," Advances in Chronic Kidney Disease, vol. 22, no. 2, pp. e1-e6, 2015.
[62] C.-C. Yu, A. Fornoni, A. Weins et al., "Abatacept in B7-1positive proteinuric kidney disease," The New England Journal of Medicine, vol. 369, no. 25, pp. 2416-2423, 2013.

[63] M. S. Joy, D. S. Gipson, L. Powell et al., "Phase 1 trial of adalimumab in Focal Segmental Glomerulosclerosis (FSGS): II. Report of the FONT (Novel Therapies for Resistant FSGS) study group," American Journal of Kidney Diseases, vol. 55, no. 1, pp. 50-60, 2010.

[64] H. Trachtman, F. C. Fervenza, D. S. Gipson et al., "A phase 1, single-dose study of fresolimumab, an anti-TGF- $\beta$ antibody, in treatment-resistant primary focal segmental glomerulosclerosis," Kidney International, vol. 79, no. 11, pp. 1236-1243, 2011. 


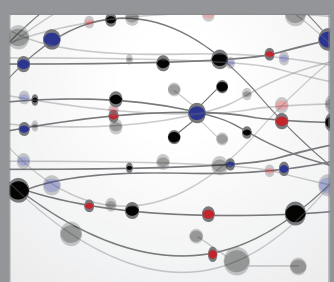

The Scientific World Journal
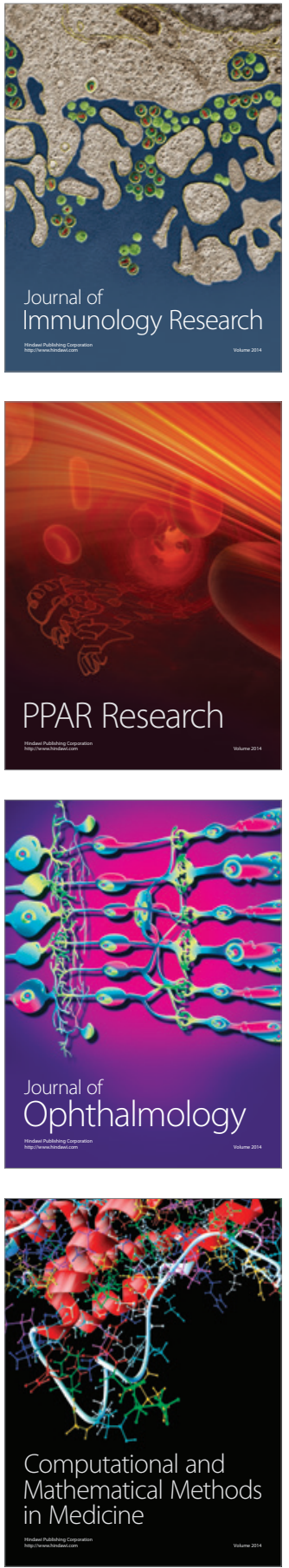

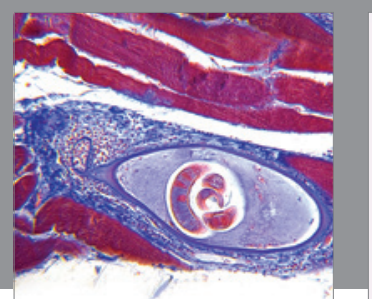

Gastroenterology Research and Practice

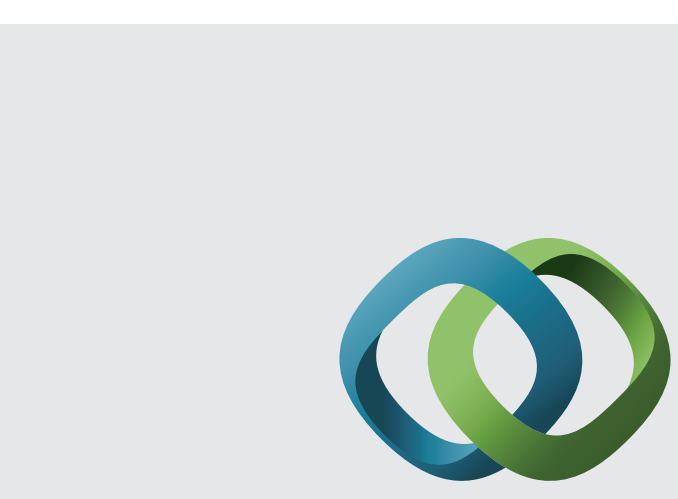

\section{Hindawi}

Submit your manuscripts at

http://www.hindawi.com
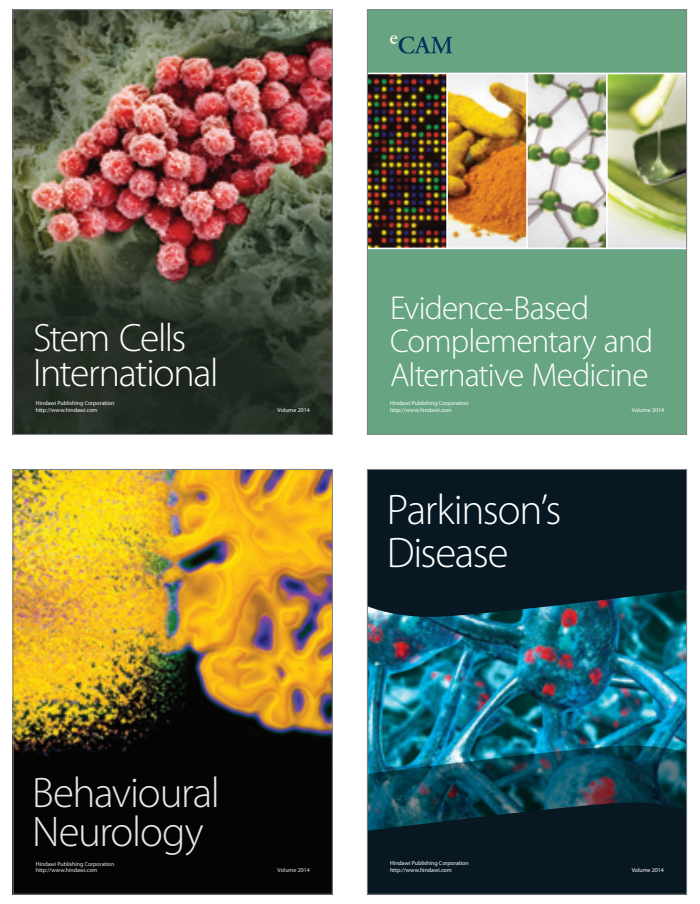
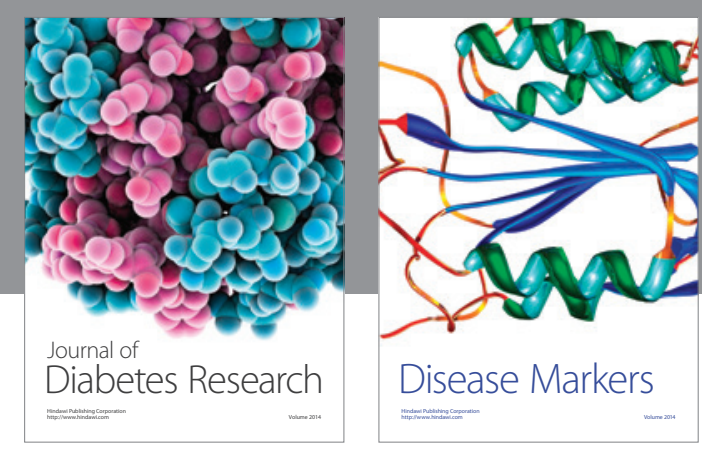

Disease Markers
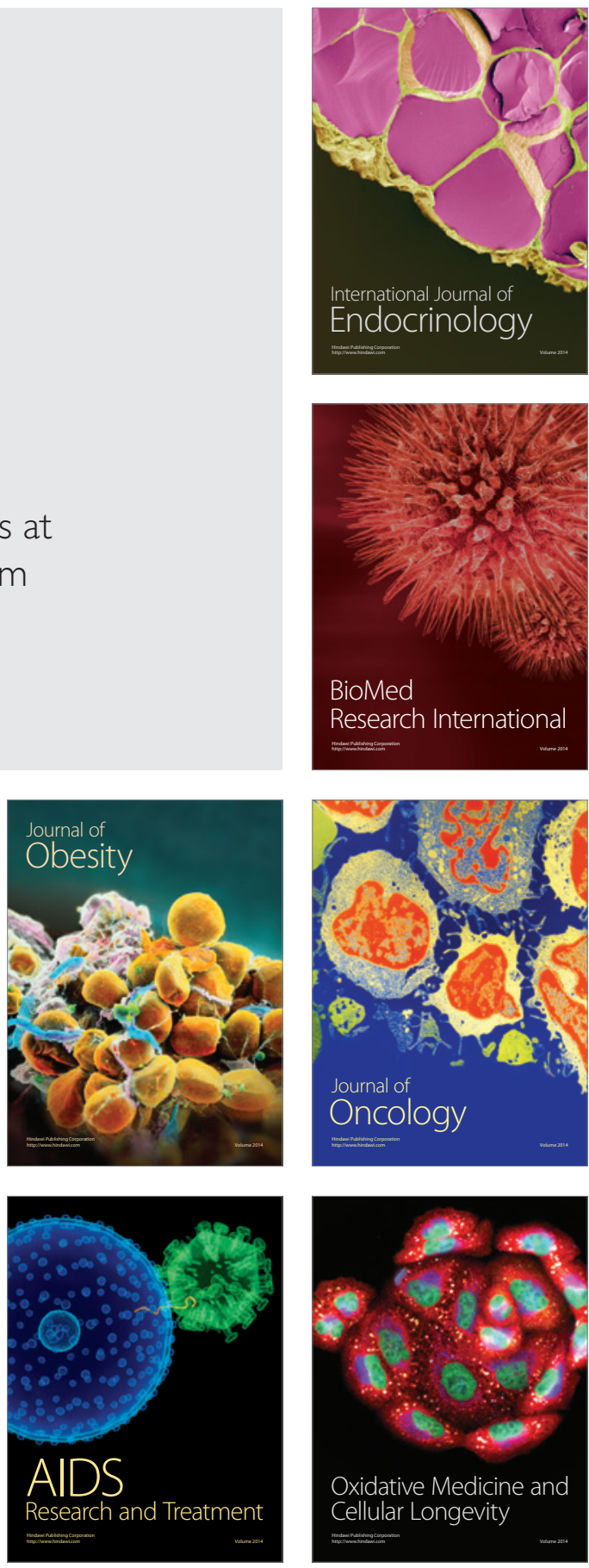